\title{
SEGURANÇA DO PACIENTE EM SERVIÇOS DE SAÚDE: UMA ANÁLISE NA CIDADE DE SALVADOR, BAHIA
}

\author{
Patient safety in health services: an analysis in the city of Salvador, Bahia
}

\section{Seguridad del paciente en servicios de salud: un análisis en la ciudad de Salvador, Bahia}

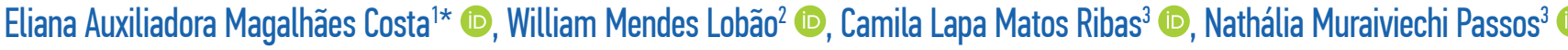

RESUMO: Objetivo: Avaliar a política nacional de segurança do paciente em hospitais de grande porte de Salvador. Método: Estudo de casos múltiplos em hospitais terciários. A coleta de dados ocorreu entre outubro de 2017 e outubro de 2018, com profissionais dos núcleos de segurança do paciente (NSP). Foram analisadas quatro variáveis independentes: constituição dos NSP; ações de planejamento do controle dos eventos adversos (EA); atividades técnico-operacionais desenvolvidas pelos NSP; e ações de monitoramento dos EA no hospital. Resultados: Dos 20 NSP existentes, estudaram-se 12 (60\%). Todos os hospitais possuem NSP constituídos, 91,7\% têm plano de segurança do paciente, e 50\% contam com profissional com dedicação exclusiva. Das instituições, 58,3\% implementam todos os protocolos obrigatórios, sendo identificação do paciente (83,3\%) e higienização das mãos (83,3\%) os mais frequentes. Os percentuais de EA identificados foram: lesão por pressão (88,9\%), queda do leito (77,8\%) e erros de medicamentos (75,0\%). Conclusão: Os EA aqui referidos sinalizam a necessidade de adequações em prol da segurança do paciente. Os NSP não atendem totalmente às políticas regulatórias vigentes no país, carecendo, portanto, de adequações e de controle sanitário efetivo.

Palavras-chave: Segurança do paciente. Dano ao paciente. Legislação hospitalar. Garantia da qualidade dos cuidados de saúde.

ABSTRACT: Objective: To evaluate the national policy of patient safety in large hospitals in Salvador. Method: Study of multiple cases in tertiary hospitals. Data collection occurred between October 2017 and October 2018, with professionals from a Center for Patient Safety (CPS). Four independent variables were analyzed: composition of CPS; action plan for management of adverse events (AE); technical and operational activities developed by the CPS; and monitoring of AE in the hospital. Results: In a sample of 20 CPS, 12 (60\%) were studied. All hospitals have a CPS established, $91.7 \%$ have patient safety plan, and 50\% have a professional exclusively dedicated to it. Among the institutions, $58.3 \%$ implement all required protocols. The most frequent are patient identification (83.3\%) and hand hygiene (83.3\%). Percentages of AE identified were: pressure wound (88.9\%), bed fall (77.8\%) and medication errors (75.0\%). Conclusion: The AE mentioned here signal the need for adjustments for patient safety. The CPS do not fully meet Brazil's current regulatory policies, thus lacking adequacies and effective sanitary control.

Keywords: Patient safety. Patient harm. Hospital legislation. Healthcare quality assurance.

RESUMEN: Objetivo: evaluar la política nacional de seguridad del paciente en grandes hospitales de Salvador. Método: estudio de caso múltiple en hospitales terciarios. La recopilación de datos tuvo lugar entre octubre de 2017 y octubre de 2018, con profesionales de los núcleos de seguridad del paciente (NSP). Se analizaron cuatro variables independientes: constitución de la NSP; planificación de acciones para controlar eventos adversos (EA); actividades técnico-operativas desarrolladas por NSP; y acciones de monitoreo de AE en el hospital. Resultados: De los 20 NSP existentes, 12 (60\%) fueron estudiados. Todos los hospitales tienen un NSP establecido, el 91.7\% tiene un plan de seguridad del paciente y el 50\% tiene un profesional con dedicación exclusiva. De las instituciones, el 58.3\% implementa todos los protocolos obligatorios, siendo la identificación del paciente (83.3\%) y la higiene de las manos (83.3\%) las más frecuentes. Los porcentajes de EA identificados fueron: lesión por presión (88.9\%), caída de la cama (77.8\%) y errores de medicación (75.0\%). Conclusión: El AE al que se hace referencia aquí indica la necesidad de ajustes a favor de la seguridad del paciente. Los NSP no cumplen totalmente con las políticas regulatorias vigentes en el país, por lo tanto, necesitan ajustes y un control sanitario efectivo.

Palabras clave: Seguridad del paciente. Daño del paciente. Legislación hospitalaria. Garantía de la calidad de atención de salud.

'Doutora em Saúde Pública. Professora adjunta da Universidade do Estado da Bahia (UNEB) - Salvador (BA), Brasil.

2Enfermeiro; doutor em Biotecnologia em Saúde e Medicina Investigativa. Professor assistente da UNEB - Salvador (BA), Brasil.

${ }^{3}$ Discente do curso de Enfermagem da UNEB - Salvador (BA), Brasil.

*Autora correspondente: costaeliana2003@hotmail.com

Recebido: 04/05/2018 - Aprovado: 23/12/2019

DOI: $10.5327 / Z 1414-4425202000010004$ 


\section{INTRODUÇÃO}

Os avanços tecnológicos incorporados aos cuidados à saúde têm possibilitado benefícios para a restauração da saúde e o prolongamento da vida, entretanto a utilização dessas tecnologias trouxe, também, sérios riscos para o paciente, suscitando questões teórico-práticas de segurança e efetividade dos processos. Nesse contexto, milhares de pacientes sofrem danos decorrentes de erros diagnósticos e terapêuticos ocorridos durante os cuidados com a saúde, e o papel dos hospitais, das agências reguladoras e de gestores dos serviços de saúde tem sido amplamente discutido, bem como a identificação dos fatores de risco que comprometem a segurança do paciente ${ }^{1-3}$.

Reconhecendo que a segurança do paciente é um problema de saúde pública de âmbito global, a Organização Mundial da Saúde (OMS) lançou em 2004 a Aliança Mundial para a Segurança do Paciente, com o propósito de desenvolver normas e padrões universais para a promoção de políticas baseadas em evidências, prestação de assistência a países em várias áreas-chave e contribuição para uma agenda mundial para a pesquisa nessa área ${ }^{4}$. Para tanto, essa aliança lançou três desafios globais: o primeiro em 2005, com foco na prevenção das infecções relacionadas à assistência à saúde (IRAS); o segundo, em 2008, voltado à segurança na realização de procedimentos cirúrgicos 5 ; e o terceiro, lançado em 2017, com o objetivo de reduzir o nível de danos graves evitáveis associados a medicamentos ${ }^{6}$.

No Brasil, o Ministério da Saúde (MS) e a Agência Nacional de Vigilância Sanitária (ANVISA), atendendo aos desafios globais da OMS, lançaram em 2013 a Portaria n ${ }^{\circ} 529 / 2013^{7}$ e a Resolução de Diretoria Colegiada (RDC) no $36 / 2013^{8}$, que instituem, respectivamente, o Programa Nacional de Segurança do Paciente (PNSP) e as ações para a segurança do paciente em serviços de saúde.

Posteriormente, o MS publicou as portarias $\mathrm{n}^{\circ} 1.377 \mathrm{e}$ $\mathrm{n}^{\mathrm{o}} 2.095$, de $2013^{9,10}$, que aprovam os protocolos básicos de segurança do paciente a serem implantados nos hospitais brasileiros:

- Cirurgia segura;

- Prática de higienização das mãos em serviços de saúde;

- Prevenção de úlceras por pressão;

- Prevenção de quedas em pacientes internados;

- Identificação do paciente;

- Segurança na prescrição, no uso e na administração de medicamentos.
Com essas portarias, tornou-se obrigatória, em todos os serviços de saúde do país, a constituição de núcleos de segurança do paciente (NSP), com os objetivos de definir e implementar um plano de segurança do paciente (PSP), segundo a necessidade e as especificidades do serviço.

Para além das questões normativas, a construção de uma cultura de segurança do paciente enquanto pilar estrutural dos serviços de saúde deve favorecer a implantação de práticas seguras que busquem o aperfeiçoamento de processos organizacionais, com os propósitos de reduzir a incidência de eventos adversos (EA) e promover a melhoria contínua da qualidade da assistência ${ }^{11}$.

Nesse sentido, este estudo procura responder à seguinte questão central: como os hospitais da cidade de Salvador estão implementando a PNSP? Essa pergunta central desdobra-se em outras perguntas subjacentes: como estão constituídos os NSP nesses hospitais? Quais são os eventos adversos mais frequentes nos hospitais da capital da Bahia?

\section{OBJETIVO}

Avaliar a implementação da PNSP por meio dos NSP dos hospitais da cidade de Salvador, tendo em vista a proteção da saúde dos pacientes internados nas instituições estudadas.

\section{MÉTODO}

Trata-se de um estudo de avaliação, descritivo e de casos múltiplos, cuja unidade de análise foi a implementação das ações dos NSP dos hospitais avaliados e intitulados nessa metodologia de $\operatorname{casos}^{12}$.

O projeto de pesquisa foi aprovado pelo Comitê de Ética em Pesquisa Envolvendo Seres Humanos da Universidade do Estado da Bahia (UNEB), mediante inscrição na Plataforma Brasil (Certificado de Apresentação para Apreciação Ética CAAE: 84683315.0.0000.0057), parecer final 2.574.463, e recebeu apoio financeiro do Conselho Nacional de Desenvolvimento Científico e Tecnológico (CNPQ) (processo 400316/2016-1).

Participaram do estudo os hospitais públicos e privados de grande porte (número de leitos maior do que 150) localizados na região metropolitana de Salvador (BA), selecionados por meio dos dados da Secretaria da Saúde do Estado da Bahia, com identificação de 20 hospitais. Optou-se por estudar esses hospitais por assistirem um grande número de pacientes nas mais diversas especialidades, resultando num 
maior nível de complexidade assistencial, bem como pela alta rotatividade de pacientes e profissionais ${ }^{13-15}$, o que pode implicar aumento da possibilidade da ocorrência de EA nos pacientes internados nessas instituições.

Após a identificação, os hospitais foram contatados por meio telefônico para que se expusessem os objetivos da pesquisa, se obtivesse permissão para sua realização e se agendasse a coleta de dados, feita presencialmente nos meses de dezembro de 2017 a outubro de 2018.

A coleta de dados constou de uma entrevista com o profissional responsável pelo NSP de cada hospital, por intermédio de um formulário semiestruturado elaborado pelos autores, e deu-se por duas bolsistas do curso de Enfermagem da UNEB, devidamente treinadas e supervisionadas para essa atividade.

Quatro variáveis independentes foram analisadas:

- Constituição do NSP e sua estrutura organofuncional (infraestrutura relacionada a recursos humanos; profissional responsável e outros profissionais; recursos, equipamentos, insumos, materiais destinados ao NSP);

- Ações de planejamento do controle dos EA no serviço de saúde (SS) (PSP; protocolos de segurança adotados);

- Atividades técnico-operacionais desenvolvidas pelo NSP;
- Ações de monitoramento dos eventos adversos no hospital.

Os dados coletados foram tabulados pelo software EpiData ${ }^{\circledR}$ versão 3.1, e as análises estatísticas, realizadas pelo pacote estatístico STATA ${ }^{\circledR}$ versão 12.

\section{RESULTADOS}

Dos 20 hospitais que atenderam ao critério de inclusão, oito (40\%) não autorizaram a coleta de dados por diversas razões, o que resultou em 12 (60\%) instituições integrantes deste estudo.

Todos os hospitais participantes são de grande porte (Tabela 1), com média de 376 leitos (variação $=150-955$; desvio padrão $\mathrm{DP}=67,07)$, e três $(25 \%)$ são hospitais-escola. Em relação à entidade mantenedora, cinco (41,7\%) são públicos, quatro $(33,3 \%)$ são filantrópicos, dois (16,7\%) privados e um $(8,3 \%)$ de gestão público-privada. Os hospitais participantes possuem, em média, quatro unidades de terapia intensiva (variação $=1-7 ; \mathrm{DP}=2,16$ ), com média de 68,4 leitos (variação=20-124; $D P=37,2$ ).

Tabela 1. Caracterização dos hospitais participantes $(n=12)^{*}$.

\begin{tabular}{|c|c|c|c|c|c|}
\hline Entidade mantenedora & n (12) & $\%$ & Especialidades & n (12) & $\%$ \\
\hline Pública & 5 & 41,7 & Clínica médica & 11 & 91,7 \\
\hline Filantrópica & 4 & 33,3 & Clínica cirúrgica & 10 & 83,3 \\
\hline Privada & 2 & 16,7 & Geral & 5 & 41,7 \\
\hline Parceria público-privada & 1 & 8,3 & Pediatria & 5 & 41,7 \\
\hline Perfil de atendimento & n (12) & $\%$ & Ortopedia & 5 & 41,7 \\
\hline Adulto & 11 & 91,7 & Cardiologia & 4 & 33,3 \\
\hline Pediatria & 8 & 66,7 & Urologia & 4 & 33,3 \\
\hline Neonatologia & 4 & 33,3 & Angiologia/cirurgia vascular & 4 & 33,3 \\
\hline Hospital-escola & n (12) & $\%$ & Neurologia & 3 & 25 \\
\hline Sim & 3 & 25 & Gastroenterologia & 3 & 25 \\
\hline Serviços adicionais & n (12) & $\%$ & Neonatologia & 3 & 25 \\
\hline Serviços ambulatoriais & 11 & 91,7 & Nefrologia & 2 & 16,7 \\
\hline Hemodiálise & 9 & 75 & Neurocirurgia & 1 & 8,3 \\
\hline Hemodinâmica & 7 & 58,3 & Outras especialidades & 8 & 66,7 \\
\hline Sistema de notificação & n (12) & $\%$ & Estatística descritiva & Média & DP \\
\hline Eletrônico & 7 & 63,6 & Leitos (150-955) & 376,01 & 67,07 \\
\hline Manual & 4 & 36,4 & Número de UTI (1-7) & 4 & 2,16 \\
\hline Inexistente & 1 & 8,3 & Leitos de UTI (20-124) & 64,9 & 37,2 \\
\hline
\end{tabular}

*Pode variar de acordo com o número de missing; UTI: unidade de terapia intensiva; DP: desvio padrão. 
Essas instituições são majoritariamente destinadas à população adulta (11 casos/91,7\%), pediátrica (oito/66,7\%) e neonatológica (quatro $/ 33,3 \%$ ) e atendem a diversas especialidades médicas, destacando-se clínica médica $(11 / 91,7 \%)$ e cirúrgica (10/83,3\%), geral (cinco / 41,7\%), pediatria (cinco/41,7\%) e ortopedia (cinco/ $41,7 \%$ ). Além da assistência hospitalar, essas instituições também disponibilizam atendimento ambulatorial (11/91,7\%), hemodiálise (nove/ $75 \%$ ) e hemodinâmica (sete/58,3\%).

Quanto à notificação de incidentes envolvendo pacientes, em quatro hospitais $(36,4 \%)$ essa tarefa ainda é realizada de forma manual, em sete casos $(63,6 \%)$ ocorre por meio de um sistema informatizado, e em um caso (5\%) não existe sistema de notificação de EA.

Na Tabela 2, descrevemos a estrutura organofuncional dos NSP dos hospitais estudados.

Dos doze NSP dos hospitais estudados, nove (75\%) têm uma sala exclusiva para o NSP e 11 deles $(91,7 \%)$ contam com auxílio de computadores. Onze NSP $(91,7 \%)$ possuem insumos e recursos materiais, entretanto apenas quatro $(33,3 \%)$ dispõem de recursos financeiros próprios para o desenvolvimento das atividades de segurança.

Esses núcleos foram formalmente constituídos pela alta administração do hospital em todos os casos, dos quais cinco $(41,7 \%)$ estão inseridos no serviço de qualidade, quatro $(33,3 \%)$ estão organizados como serviços autônomos e três NSP (25\%) estão em outras inserções organizacionais de caráter consultivo.

A existência de um profissional responsável e com dedicação exclusiva para o NSP, conforme recomendado por normativa legal ${ }^{7}$, foi identificada em apenas seis hospitais (50\%).

Em relação ao período de implantação, dez NSP (90,9\%) foram implementados em 2013, em atendimento às normativas nacionais, e um $(9,1 \%)$ antes dessa obrigatoriedade. Na maioria dos hospitais (sete / 63,6\%), o sistema interno de notificações de incidentes foi adotado após 2013.

Quase todos os NSP (11/91,7\%) contam com PSP específico para a instituição: de sete PSP $(58,3 \%)$ constam estratégias para estimular a participação do paciente e dos familiares na assistência prestada; em nove PSP (75\%) estão presentes estratégias para a promoção da segurança nas terapias nutricionais enteral e parenteral; e em dez $(83,3 \%)$ há estratégias para promoção da segurança na prescrição, no uso e na administração de sangue e hemocomponentes.

Entre os profissionais que atuam nos NSP estudados, identificou-se que os enfermeiros fazem parte de todos os núcleos (12/100\%), os médicos de 11 (91,7\%), farmacêuticos de dez
Tabela 2. Estrutura organofuncional dos núcleos de segurança do paciente (NSP) dos hospitais estudados $(n=12)^{*}$.

\begin{tabular}{|c|c|c|}
\hline Característica & $\mathbf{n}$ & $\%$ \\
\hline Sala exclusiva para o NSP $(n=12)$ & 9 & 75 \\
\hline Computador ( $n=12)$ & 11 & 91,7 \\
\hline $\begin{array}{l}\text { Existência de recursos financeiros próprios } \\
(n=12)\end{array}$ & 4 & 33,3 \\
\hline $\begin{array}{l}\text { Existência de insumos e materiais para o } \\
\text { desenvolvimento das atividades }(n=12)\end{array}$ & 11 & 91, \\
\hline NSP formalmente constituído $(n=12)$ & 12 & 100 \\
\hline NSP inserido no serviço de qualidade $(n=12)$ & 5 & 41,7 \\
\hline NSP como serviço autônomo ( $n=12)$ & 4 & \\
\hline $\begin{array}{l}\text { NSP com outras inserções de caráter } \\
\text { consultivo }\end{array}$ & 3 & \\
\hline Profissional responsável exclusivo ( $n=12)$ & 6 & 50 \\
\hline \multicolumn{3}{|c|}{ Ano de implantação do núcleo de segurança do paciente $(n=11)$} \\
\hline 2011 & 1 & 9,1 \\
\hline 2013 & 2 & 18,2 \\
\hline 2014 & 1 & 9,1 \\
\hline 2015 & 3 & 27,3 \\
\hline 2016 & 2 & 18,2 \\
\hline 2017 & 1 & 9,1 \\
\hline 2018 & 1 & 9, \\
\hline
\end{tabular}

Ano de implantação do sistema interno de notificação de incidentes $(n=11)$

\begin{tabular}{|l|c|c|}
\hline 2005 & 1 & 9,1 \\
\hline 2010 & 2 & 18,2 \\
\hline 2012 & 1 & 9,1 \\
\hline 2014 & 1 & 9,1 \\
\hline 2015 & 1 & 9,1 \\
\hline 2016 & 2 & 18,2 \\
\hline 2017 & 3 & 27,3 \\
\hline 2018 & 1 & 9,1 \\
\hline $\begin{array}{l}\text { Existe planejamento escrito e disponível no } \\
\text { NSP com metas específicas para o controle dos }\end{array}$ & 11 & 91,7 \\
\hline $\begin{array}{l}\text { ventos adversos no hospital (n=12) } \\
\begin{array}{l}\text { PSP apresenta estratégias para estimular } \\
\text { participação do paciente e dos familiares na }\end{array}\end{array}$ & 7 & 58,3 \\
\hline $\begin{array}{l}\text { sssistência prestada (n=12) } \\
\text { O PSP apresenta estratégias para promoção da } \\
\text { segurança nas terapias nutricionais enteral e }\end{array}$ & 9 & 75 \\
\hline $\begin{array}{l}\text { parenteral (n=12) } \\
\text { O PSP apresenta estratégias para promoção }\end{array}$ & & \\
\hline $\begin{array}{l}\text { da segurança na prescrição, no uso e na } \\
\text { dministração de sangue e hemocomponentes } \\
\text { (n=12) }\end{array}$ & 10 & 83,3 \\
\hline
\end{tabular}

*Pode variar de acordo com o número de missing, por conta do número de NSP que nã forneceram essa informação; PSP: plano de segurança do paciente. 
$(83,3 \%)$ e outras categorias profissionais de nove $(75,0 \%)$ instituições. A maioria dos profissionais que trabalham nesses núcleos não possui capacitação específica para essa área de atuação, embora três deles tenham especialização em segurança do paciente e um em gestão hospitalar.

Os protocolos básicos de segurança do paciente recomendados pelo MS e implementados pelos NSP dos hospitais estudados estão descritos na Tabela 3.

Dos 12 NSP estudados, somente sete $(58,3 \%)$ implementavam todos os seis protocolos básicos recomendados pelo MS, e dois NSP (16,7\%) não praticavam nenhum deles. Os protocolos mais adotados são: identificação do paciente e higienização das mãos (83,3\%); cirurgia segura e prevenção de lesão por pressão (75\%). Os protocolos menos implementados são: prevenção de erros na administração de medicamentos e prevenção de quedas (66,7\%).

Identificou-se que, além dos protocolos obrigatórios, oito NSP (66,7\%) executavam protocolos adicionais, a exemplo dos bundles de cateter venoso central, prevenção de tromboembolismo venoso (três/37,5\%), sepse e broncoaspiração (dois $/ 25 \%)$.

As atividades técnico-operacionais desenvolvidas pelos NSP estão descritas na Tabela 4.

Em relação ao treinamento da equipe multidisciplinar de saúde acerca da temática da segurança do paciente, todos os NSP realizam essa atividade e têm registro dessa ação. Para tanto, utilizam várias estratégias de comunicação, que

Tabela 3. Protocolos de segurança do paciente implementados pelos núcleos de segurança dos hospitais estudados $(n=12)$.

\begin{tabular}{|l|c|c|}
\hline Número de protocolos implementados & $\mathbf{n}$ & $\%$ \\
\hline Nenhum & 2 & 16,7 \\
\hline Três & 1 & 8,3 \\
\hline Quatro & 1 & 8,3 \\
\hline Cinco & 1 & 8,3 \\
\hline Seis & 7 & 58,3 \\
\hline Protocolos básicos implementados & $\mathbf{n}$ & $\%$ \\
\hline Identificação do paciente & 10 & 83,3 \\
\hline Higienização das mãos & 10 & 83,3 \\
\hline Cirurgia segura & 9 & 75,0 \\
\hline Prevenção de lesão por pressão & 9 & 75,0 \\
\hline Prevenção de erros de medicação & 8 & 66,7 \\
\hline Prevenção de quedas & 8 & 66,7 \\
\hline Outros protocolos & 8 & 66,7 \\
\hline
\end{tabular}

envolvem campanhas pontuais, elaboração de mandalas com passos sobre segurança do paciente, painéis e alertas, roletas educativas e seminários teóricos anuais.

Entre as atividades técnico-operacionais desenvolvidas pelos NSP estudados, identificou-se que $100 \%$ analisavam os dados sobre os incidentes e EA no hospital; e 11 (91,7\%) estimulavam a notificação de incidentes pelos colaboradores do hospital e realizavam o monitoramento dos indicadores dos protocolos de SP.

Dos NSP participantes, dez (83,3\%) acompanham as ações descritas no PSP, compartilham e divulgam os dados sobre os EA ocorridos no hospital, entretanto a notificação ao Sistema Nacional de Vigilância Sanitária dos EA ocorridos no hospital até o $15^{\circ}$ dia útil ocorre em nove NSP (75\%), e a notificação em até 72 horas dos EA que evoluíram para óbito só foi identificada em seis (50\%) dos oito NSP que fornecem essa informação.

Os principais incidentes e EA ocorridos nos hospitais de grande porte de Salvador referidos pelos NSP estudados estão descritos na Tabela 5.

Somente nove NSP (75\%) forneceram as informações para a análise da distribuição percentual dos incidentes e EA ocorridos nos hospitais de grande porte de Salvador. Os EA

Tabela 4. Atividades técnico-operacionais desenvolvidas pelos núcleos de segurança do paciente (NSP) dos hospitais estudados $(n=12)$.

\begin{tabular}{|c|c|c|}
\hline $\begin{array}{l}\text { Atividades técnico-operacionais } \\
\text { desenvolvidas pelos NSP }\end{array}$ & $\mathbf{n}$ & $\%$ \\
\hline $\begin{array}{l}\text { Realização de programas de treinamento } \\
\text { acerca da SP para profissionais de saúde }\end{array}$ & 12 & 100 \\
\hline $\begin{array}{l}\text { Análise e avaliação dos dados sobre } \\
\text { incidentes e EA no hospital }\end{array}$ & 12 & 100 \\
\hline $\begin{array}{l}\text { Estímulo à notificação de incidentes pelos } \\
\text { colaboradores }\end{array}$ & 11 & 91,7 \\
\hline $\begin{array}{l}\text { Realização de monitoramento dos } \\
\text { indicadores dos protocolos de SP }\end{array}$ & 11 & 91,7 \\
\hline Acompanhamento das ações descritas no PSP & 10 & 83,3 \\
\hline $\begin{array}{l}\text { Compartilhamento e divulgação de dados } \\
\text { sobre os EA ocorridos no hospital }\end{array}$ & 10 & 83,3 \\
\hline $\begin{array}{l}\text { Notificação ao Sistema Nacional de } \\
\text { Vigilância Sanitária dos EA ocorridos no } \\
\text { hospital até o } 15^{\circ} \text { dia útil }\end{array}$ & 9 & 75 \\
\hline $\begin{array}{l}\text { Notificação de óbitos ao Sistema Nacional de } \\
\text { Vigilância Sanitária em até } 72 \text { horas após os EA }\end{array}$ & 6 & 50 \\
\hline Realização de outras atividades & $10(83,3)$ & \\
\hline
\end{tabular}

SP: segurança do paciente; EA: evento adverso; PSP: programa de segurança do paciente. 
Tabela 5. Distribuição percentual dos incidentes e eventos adversos ocorridos em hospitais de grande porte de Salvador, BA $(n=9)^{*}$.

\begin{tabular}{|l|c|c|}
\hline Evento adverso & $\mathbf{n}$ & $\%$ \\
\hline Lesão por pressão $(n=9)$ & 8 & 88,9 \\
\hline Queda do leito $(\mathrm{n}=9)$ & 7 & 77,8 \\
\hline Erros de medicamentos $(\mathrm{n}=8)$ & 6 & 75 \\
\hline Flebite $(\mathrm{n}=8)$ & 4 & 50 \\
\hline Retirada acidental de drenos e tubos $(\mathrm{n}=7)$ & 3 & 42,9 \\
\hline Identificação do paciente $(\mathrm{n}=9)$ & 3 & 33,3 \\
\hline Não higienização das mãos $(\mathrm{n}=8)$ & 2 & 25 \\
\hline Erros em cirurgia $(\mathrm{n}=8)$ & 0 & 0 \\
\hline Outros eventos adversos $(n=8)$ & 4 & 50 \\
\hline
\end{tabular}

*Pode variar de acordo com o número de missing, por conta do número de núcleos de segurança do paciente que não forneceram essa informação.

e incidentes relatados pelos NSP como os mais prevalentes foram: lesão por pressão $(88,9 \%)$, queda do leito $(77,8 \%)$, erros de medicamentos $(75 \%)$, flebite $(50 \%)$, retirada acidental de drenos e tubos (42,9\%), erros na identificação do paciente (33,3\%), ausência de higienização das mãos durante o cuidado assistencial (25,0\%) e outros EA (50\%), relatados como: infecção relacionada à assistência, lesão de pele causada por oxímetro, perda de sonda vesical e trocas de dieta. Não houve notificação de EA ao longo da realização de procedimentos cirúrgicos entre os NSP que responderam a esse item da pesquisa.

\section{DISCUSSÃO}

Dos 20 NSP dos hospitais de grande porte da cidade de Salvador, estudaram-se 12 deles (60\%), na grande maioria inseridos em instituições públicas, filantrópicas e privadas, sendo três (25\%) em hospitais de ensino, contemplando, portanto, NSP em instituições com distintas formas de gestão organizacional.

Todos os núcleos estão constituídos formalmente, e a maioria possui um PSP elaborado segundo as especificidades de cada instituição, situação que denota a institucionalidade desses serviços dentro dos hospitais estudados, bem como preocupação com o planejamento de suas atividades.

A maioria dos NSP foi implantada após 2013, supostamente em atendimento às exigências definidas nos marcos regulatórios sobre segurança do paciente no país ${ }^{7-10}$, entretanto identificamos uma instituição cujo NSP foi montado antes da data do lançamento do PNSP, apontando que a temática da segurança e do gerenciamento do risco já fazia parte da agenda dessa organização, independentemente da obrigatoriedade exigida pelas normativas vigentes.

Identificou-se que os NSP atuam com distintas modalidades executivas, seja como serviço autônomo dentro das instituições hospitalares, seja inseridos nos serviços de qualidade, como órgãos consultivos, todos em conformidade com a resolução da ANVISA que recomenda que "a direção do serviço de saúde pode utilizar a estrutura de comitês, comissões, gerências, coordenações ou núcleos já existentes para o desempenho das atribuições do NSP".

Apenas metade dos NSP estudados conta com um profissional responsável e exclusivo para as atividades de segurança do paciente, situação que contraria a norma regulatória e que desfavorece a implantação dos processos de trabalho em prol da prevenção de erros em saúde nesses serviços, na medida em que na metade desses núcleos os profissionais atuam em outros serviços e respondem a eles, fragmentando a atividade de segurança do paciente. Além disso, a maioria dos profissionais que trabalham nesses núcleos não possui capacitação específica na área da segurança do paciente, o que pode ser um elemento dificultador para a sua atuação. Esses achados podem desafiar a implantação de uma cultura de segurança nessas organizações, liderada pelos NSP e definida como padrões de comportamento individuais e de grupo, os quais determinam o compromisso, o estilo e a proficiência da administração de uma organização saudável e segura ${ }^{2,3,11,16}$.

A despeito dessas limitações, todos os NSP estudados analisam os dados sobre os incidentes e EA no hospital e 91,7\% estimulam a notificação de incidentes pelos colaboradores dos hospitais, instituindo, desse modo, uma cultura da quebra do medo do registro e da informação do erro em saúde, em consonância com a compreensão do caráter multifatorial dos erros assistenciais, cuja premissa é de que os seres humanos cometem falhas e que os erros são consequências e não causas. Afinal, tem-se o conhecimento de que os principais fatores que contribuem para a ocorrência dos EA são as deficiências do sistema de prestação dos cuidados em saúde, tanto em sua concepção quanto na sua organização e no seu funcionamento ${ }^{16}$.

A notificação dos erros e incidentes que causam ou não lesão ao paciente é o elemento norteador de um programa de segurança em saúde, uma vez que o conhecimento dos erros propicia a delimitação da magnitude desses eventos na organização, além da elaboração de indicadores e tomada de decisão. Um indicador de qualidade é definido como uma 
medida quantitativa sobre algum aspecto do cuidado do paciente, e a inclusão desses indicadores pelos NSP representa uma importante estratégia para a promoção da segurança do paciente hospitalizado ${ }^{17}$.

Em relação à notificação ao Sistema Nacional de Vigilância Sanitária dos EA ocorridos no hospital até o $15^{\circ}$ dia útil do mês subsequente, identificamos que a maioria dos NSP atende a essa recomendação, embora alguns a fazem em até 72 horas, quando os EA evoluem para óbito, omitindo da ANVISA, em tempo real, os eventos mais graves ocorridos na instituição, bem como impossibilitando a participação dessa agência reguladora no acompanhamento das ações propostas pelos hospitais para a elucidação e proposição de estratégias para a prevenção de eventos mais graves.

Dos protocolos básicos recomendados pelo MS, somente sete NSP (58,3\%) implementam todos eles; cinco (41,6\%), apenas alguns; e dois núcleos (16,7\%) não adotam nenhum dos protocolos obrigatórios, configurando não conformidade sanitária e descumprimento dos planos de segurança do paciente, já que esses protocolos são essenciais para a sustentação mínima de um programa e de uma cultura de segurança institucional.

Ademais, a falta de adesão aos protocolos obrigatórios de segurança do paciente em hospitais com perfis complexos como os aqui estudados, que prestam assistência a pacientes em condições clínicas mais graves, submetidos a múltiplas intervenções e, portanto, mais propensos a sofrer efeitos indesejados do cuidado ofertado, evidencia a lacuna do controle sanitário dessas instituições de saúde a ser exercido pela vigilância sanitária.

Nesse sentido, a vigilância sanitária, órgão do sistema de saúde nacional cujo conceito constitucional é "controlar riscos relacionados aos produtos, processos e serviços de interesse à saúde humana"», é, assim, responsável pela fiscalização dos NSP segundo consta da resolução em vigor ${ }^{8}$ e, nesse exercício, pode ser um elemento catalisador para o êxito da implementação da política de segurança do paciente no país.

Os protocolos de segurança mais implementados foram os de identificação do paciente e de higienização das mãos, seguidos pelos protocolos de cirurgia segura e prevenção de lesão por pressão, e os menos implementados foram os de prevenção de erros na administração de medicamentos e prevenção de quedas. Tais protocolos, definidos como básicos, são pilares da qualidade assistencial para qualquer serviço de saúde, e sua implementação interfere diretamente nos indicadores de saúde, como taxas de permanência, morbidade e mortalidade hospitalar.
Os incidentes e EA mais prevalentes relatados pelos NSP nos hospitais pesquisados foram lesão por pressão, queda do leito e erros de medicamentos. Viram-se, também, a ocorrência de flebites, bem como de erros na retirada de drenos e tubos, na identificação do paciente e ausência de higienização das mãos. Não foram reportados EA relacionados aos procedimentos cirúrgicos.

Os EA aqui reconhecidos são, a priori, passíveis de serem previstos e diferem dos dados da literatura que reportam que, quanto à natureza, os EA de maior incidência são os relacionados à cirurgia, seguidos dos relativos a medicamentos, diagnóstico, terapêutica, procedimentos clínicos e queda $a^{18-20}$.

Os percentuais identificados de lesão por pressão, queda no leito, retirada acidental de drenos e erros de medicação sinalizam que esforços maiores dos processos de trabalho em prol da segurança do paciente se fazem necessários nessas instituições. Adicionalmente, chama a atenção o percentual de erros associados à identificação do paciente e à técnica de higienização das mãos, uma vez que os protocolos mais implementados pelos NSP aqui estudados são os de identificação do paciente e higienização das mãos (ambos 83,3\%), apontando que não basta implementar os protocolos; é preciso realizar monitoramento das práticas relacionadas.

\section{CONCLUSÃO}

Os dados deste estudo permitiram analisar, na terceira maior capital do país, a implementação da PNSP, instituída em 2013, e contribuem como parâmetro de avaliação da adesão dessa normativa para os órgãos reguladores e fiscalizadores.

A amostra, de $60 \%$ dos NSP dos hospitais investigados, constitui uma limitação e uma oportunidade para estudos futuros de seguimento.

Identificou-se que todos os hospitais de grande porte estudados respondem positivamente à PNSP. Os NSP examinados desenvolvem suas atividades de modo planejado, e a maioria implementa os protocolos básicos de segurança recomendados no país, bem como outras atividades relacionadas à segurança do paciente.

Os percentuais de EA encontrados pelos NSP dos hospitais pesquisados ratificam estudos anteriores que apontam que $\mathrm{O}$ Brasil possui uma das maiores frequências de EA evitáveis do mundo, sinalizando a necessidade de manutenção e intensificação dos processos de trabalho em prol da prevenção de erros em saúde, bem como a constituição de uma cultura de segurança nas organizações de saúde, principalmente nas mais complexas, como as deste trabalho. 
Observamos uma lacuna do controle sanitário a ser exercido pela vigilância sanitária em algumas instituições averiguadas e, nesse sentido, acreditamos que cabe ao Estado não apenas o imperativo de regular processos e serviços de saúde, mas a capacidade operacional de controlar o cumprimento das suas normativas.

\section{REFERÊNCIAS}

1. Flodgren G, Conterno LO, Mayhew A, Omar O, Pereira CR, Shepperd S. Interventions to improve professional adherence to guidelines for prevention of device-related infections. Cochrane Database Syst Rev [Internet]. 2013 [acessado em 26 abr. 2019];(3). Disponivel em: http:// doi.wiley.com/10.1002/14651858.CD006559.pub2

2. Pereira FGF, Matias Éo, Ceatano JÁ, Lima FET. Segurança do paciente e promoção da saúde: uma reflexão emergente. Rev Baiana Enfermagem [Internet]. 2015 [acessado em 23 abr. 2019];29(3):278. Disponível em: https://portalseer.ufba.br/index.php/enfermagem/ article/view/12205/pdf_10. http://dx.doi.org/10.18471/rbe.v29i3.12205

3. Okuyama JHH, Galvão TF, Silva MT. Healthcare professional's perception of patient safety measured by the hospital survey on patient safety culture: a systematic review and meta-analysis [Internet]. ScientificWorldJournal. 2018 [acessado em 25 abr. 2019]:1-11. Disponivel em: https://www.hindawi.com/journals/tswj/2018/9156301/

4. World Health Organization (WHO). The World Alliance for Patient Safety [Internet]. Genebra: WHO; 2004 [acessado em 26 abr. 2019]. 33 p. Disponível em: http://www.who.int/patientsafety/en/brochure_final.pdf

5. World Health Organization (WHO). WHO Patient Safety. World alliance for patient safety. The second global patient safety challenge: safe surgery saves lives [Internet]. Genebra: WHO Press; 2008 [acessado em 20 jan. 2019]. 32 p. Disponível emt: https://apps.who. int/iris/bitstream/handle/10665/70080/WHO_IER_PSP_2008.07_eng. pdf? sequence $=1$ \&isAllowed $=\mathrm{y}$

6. World Health Organization (WHO). Global patient safety challenge: medication without harm. WHO Glob Patient Saf Chall [Internet]. 2017 [acessado em 20 jan. 2019]. 16 p. Disponível em: http://apps.who.int/iris/ bitstream/10665/255263/1/WHO-HIS-SDS-2017.6-eng.pdf?ua=1 \&ua=1

7. Brasil. Ministério da Saúde. Portaria n 482, de $1^{\circ}$ de abril de 2014 Institui o Programa Nacional de Segurança do Paciente (PNSP) [Internet]. Diário Oficial da União; 2013 [acessado em 2 dez. 2018]. p. 1-4. Disponível em: http://bvsms.saude.gov.br/bvs/saudelegis/ gm/2014/prt0482_01_04_2014.html

8. Agência Nacional de Vigilância Sanitária (ANVISA). RDC n 36 , de 25 de julho de 2013. Institui ações para a segurança do paciente em serviços de saúde e dá outras providências. Diário Oficial da União; 2013.

9. Brasil. Ministério da Saúde. Portaria no 1.377, de 9 de julho de 2013. Aprova os Protocolos de Segurança do Paciente [Internet]. Diário Oficial da União; 2013 [acessado em 2 dez. 2018]. Disponível em: http://bvsms.saude.gov.br/bvs/publicacoes/parto.pdf

10. Brasil. Ministério da Saúde. Portaria n².095, de 24 de setembro de 2013. Aprova os Protocolos Básicos de Segurança do Paciente. Diário Oficial da União; 2013.

11. Andrade LEL, Lopes JM, Souza Filho MCM, Vieira Júnior RF, Farias LPC, dos Santos CCM, et al. Cultura de segurança do paciente em três hospitais brasileiros com diferentes tipos de gestão. Ciênc Saúde Coletiva [Internet]. 2018 [acessado em 21 mar. 2019];23(1):161-72. Disponível em: http://www.scielo.br/scielo. php?script=sci_arttext\&pid=S1413-81232018000100161\&lng=pt\& tlng=pt

12. Yin RK. Estudo de caso: planejamento e Métodos. 2. ed. Porto Alegre: Bookman; 2001. 200 p.

13. Leitão IMT de A, Sousa FSP de, Santiago JC dos S, Bezerra IC, de Morais JB. Absenteísmo, rotatividade e indicadores de qualidade do cuidado em enfermagem: estudo transversal. Online Brazilian J Nurs [Internet]. 2017 [acessado em 2 dez. 2018];16(2):119-29. Disponível em: www.objnursing.uff.br/index.php/nursing/article/view/5623. https://doi.org/10.17665/1676-4285.20175623

14. Novaretti MCZ, Santos E de V, Quitério LM, Daud-Gallotti RM. Nursing workload and occurrence of incidents and adverse events in ICU patients. Rev Bras Enferm [Internet]. 2014 [acessado em 2 dez. 2018];67(5):692-9. Disponível em: https://doi.org/10.1590/0034-7167.2014670504

15. Siman AG, Brito MJM. Mudanças na prática de enfermagem para melhorar a segurança do paciente. Rev Gaúcha Enferm [Internet]. 2016 [acessado em 2 dez. 2018];37(esp.):1-9. Disponível em: http://www. scielo.br/pdf/rgenf/v37nspe/0102-6933-rgenf-1983-14472016esp68271. pdf. http://dx.doi.org/10.1590/1983-1447.2016.esp.68271

16. Reis CT, Martins M, Laguardia J. A segurança do paciente como dimensão da qualidade do cuidado de saúde: um olhar sobre a literatura. Ciênc Saúde Coletiva [Internet]. 2013;18(7):2029-36. Disponível em: http://www.scielosp.org/scielo. php?script=sci_arttext\&pid=S1413-81232013001500018

17. Brasil. Agência Nacional de Vigilância Sanitária (ANVISA). Assistência segura: uma reflexão teórica aplicada à prática [Internet]. Brasília; 2017 [acessado em 20 dez. 2018]. p. 18-27 Disponível em: http:// portal.anvisa.gov.br/documents/33852/3507912/Caderno+1++Assistência+Segura+-+Uma+Reflexão+Teórica+Aplicada+à+Prát ica/97881798-cea0-4974-9d9b-077528ea1573

18. Ortega DB, D'Innocenzo M, Silva LMG da, Bohomol E. Análise de eventos adversos em pacientes internados em unidade de terapia intensiva. Acta Paul Enferm. 2017;30(2):168-73. http://dx.doi. org/10.1590/1982-0194201700026

19. Carlesi KC, Padilha KG, Toffoletto MC, Henriquez-Roldán C, Juan MAC. Patient safety incidents and nursing workload. Rev Latino-Am Enfermagem [Internet]. 2017 [acessado em 20 dez. 2018];25. Disponível em: http://www.scielo.br/scielo.php?script=sci_ arttext\&pid=S0104-11692017000100319\&lng=en\&tlng=en. http:// dx.doi.org/10.1590/1518-8345.1280.2841

20. de Araújo IS, de Carvalho R. Eventos adversos graves em pacientes cirúrgicos: ocorrência e desfecho. Rev SOBECC. 2018;23(2):83. https:// doi.org/10.5327/Z1414-4425201800020004 